\title{
Deformations and Thermal Stability of Carbon Nanotube Ropes
}

\author{
María J. López, Angel Rubio, and Julio A. Alonso
}

\begin{abstract}
Structural and thermal characteristics of crystalline ropes of single-wall carbon nanotubes (SWCNTs) are investigated. Novel crystalline ropes of polygonized SWCNTs produced by laser irradiation exhibit rounded-hexagonal cross sections in contrast to earlier observations of circular tubes. Extensive molecular dynamics (MD) simulations lead to several metastable structures of the lattice characterized by different tube cross sections, hexagonal, rounded-hexagonal and circular, and increasing cell volume. The competition between different tube shapes is analyzed and compared to experiments. On the other hand, bundles of SWCNTs coalesce, forming multiwall carbon nanotubes under thermal treatment at high temperatures. Extensive MD simulations confirm the single-wall-to-multiwall transformation and suggest the physical patching-and-tearing mechanism underlying the concerted coalescence of the tubes.
\end{abstract}

Index Terms-Carbon nanotubes (CNTs), coalescence, deformations, ropes.

\section{INTRODUCTION}

A S IT IS WELL known, carbon nanotubes (CNTs) are seamless cylinders of $s p^{2}$ carbon atoms arranged in a graphitic honeycomb structure. CNTs may be one fold (i.e., single-wall carbon nanotubes (SWCNTs) [1]) or contain several cylinders nested one inside another (i.e., mutiple-wall carbon nanotubes (MWCNTs) [2]). SWCNTs, in many cases, self-organize into crystalline bundles [3], [4] (a set of a few to a few hundred aligned tubes arranged in a two-dimensional triangular lattice in the plane perpendicular to their common axes). Although these metastable forms of carbon are subjected to intensive investigations, there are still important gaps in our understanding and control of the properties of these materials. A thorough understanding of CNTs includes the detailed and comprehensive characterization of their possible deformations due to interactions with a substrate or with other tubes, and of their stability under thermal treatment or chemical agents.

Manuscript received June 10, 2003; revised October 30, 2003. This work was supported by the Ministerio de Ciencia y Tecnología (MCYT) of Spain under Grant MAT2002-04499-C02, by the European Community Research Training Network-COMELCAN, and by J. de Castilla y León under Grant CO01/102. The work of M. J. López was supported by the Spanish MCYT under the Ramón y Cajal Program. This paper was presented in part at the Symposium of Microtechnologies for the New Millennium, Nanotechnology Conference, May $10-21,2003$

M. J. López is with the Departamento de Física Teórica, Universidad de Valladolid, 47011 Valladolid, Spain (e-mail: maria@lab2.fam.cie.uva.es).

A. Rubio is with the Departamento de Física de Materiales, Universidad del País Vasco, 20018 San Sebastián/Donostia, Spain and also with the Donostia International Physics Center, 20018 San Sebastián/Donostia, Spain.

J. A. Alonso is with the Departamento Física Teórica Universidad de Valladolid, 47011 Valladolid, Spain and also with the Donostia International

Physics Center, 20018 San Sebastián/Donostia, Spain.

Digital Object Identifier 10.1109/TNANO.2004.828522
It has been shown that CNTs may deform elastically away from their ideal circular cross sections when they interact either with a substrate [5] or with other tubes [6], [7]. Deformations of the tubes may have nontrivial effects in their properties such as their conductivity or phonon spectrum. Tubes in a bundle interact one to another through attractive van der Waals forces, similar to the ones acting between graphene layers in graphite. Therefore, the intertube interaction could prompt elastic structural changes in the tubes. We have being able to synthesize novel crystalline bundles of "polygonized" (with hexagonal cross sections) SWCNTs [8]. Our finding opens up the question of what is the equilibrium configuration of a lattice of aligned tubes and the possibility of the existence of several metastable structures that could be obtained depending on the growth conditions. To shed some light on this problem, we have performed extensive molecular dynamic (MD) simulations of lattices of monodisperse armchair and zigzag SWCNTs as a function of tube diameter. We find several metastable structures of the lattice characterized by different tube cross sections, hexagonal, rounded-hexagonal and circular, and increasing cell volume. Our results, presented in Section III, help to interpret both the earlier (circular) and currently (hexagonal) observed tube shapes.

On the other hand, since CNTs are metastable (the most stable form of carbon is graphite), they may transform into more stable structures under the appropriate annealing conditions. We have found that bundles of SWCNTs coalesce, forming MWCNTs, containing from 2 to 6 nested tubes, under thermal treatment at high temperatures [9]-[11]. This structural transformation is confirmed by extensive MD [11] simulations, presented in Section IV. The simulations combined with the experiments suggest a "patching-and-tearing" mechanism for the SWCNT to MWCNT transformation underlying the "concerted" coalescence of the tubes that begins with their polymerization.

\section{THEORETICAL MODEL}

Due to the large number of atoms (up to 6000 nonequivalent atoms) and the large time scales (of the order of $1000 \mathrm{ps}$ ) involved in the simulation of the structural and thermal properties of SWCNT bundles, the use of accurate $a b$ initio quantum techniques in the description of these systems becomes impractical if not completely unfeasible. Therefore, we use a reliable and computational efficient many-body interatomic potential to mimic the carbon-carbon interactions. The potential consist of two terms, which are: 1) a short-range part, which is well suited for describing the intratube covalent bonds [12], [13] and 2) a 
long-range term, which describes the van der Waals interactions between adjacent tubes [14]. This potential appropriately describes both the covalent bond in diamond and graphite and the van der Waals attraction between graphene layers in graphite. It has been successfully applied to the study of fullerenes and tubes.

The dynamical simulations are performed within the constant-energy constant-volume ensemble. The time evolution of the system is obtained by numerical integration using the velocity version of the Verlet algorithm of the classical Newtonian equations of motion. To investigate possible metastable structures of the lattice of SWCNTs, a number of high-energy configurations (for several lattice parameters and various tube shapes) are generated and relaxed using the thermal quenching procedure. This procedure consists of the step-wise removal of the kinetic energy of the system, along a dynamical trajectory, until the forces on all the atoms vanish and a local minimum on the potential energy surface has been found. The lowest energy minimum found will likely correspond to the equilibrium configuration of the lattice, and several metastable structures will be also obtained. We have also investigated the thermal behavior of bundles of SWCNT heat treated at high temperatures. The simulation begins with cool tubes, which are progressively heated up by scaling up the velocities of all the atoms. The temperature of the bundles is raised up to a value $\left(3000-3500{ }^{\circ} \mathrm{C}\right)$ such that the rate of coalescence is high enough for being observed within the time scale $(t \leq 1000 \mathrm{ps})$ of our simulations.

\section{POLYGONIZED ROPES}

Novel crystalline ropes of polygonized SWCNTs have been produced by $\mathrm{CO}_{2}$ laser ablation [8]. The ropes consist of monodisperse SWCNTs with tube diameters $D$ of approximately $17 \AA$ arranged in a triangular lattice. High-resolution transmission electron microscopy (HRTEM) images of the lattice [8] clearly show the departure of the tubes from the circular cross section and the appearance of facets, parallel to one another, between adjacent tubes. The polygonized hexagonal cross section of the tubes is compatible with the two-dimensional triangular symmetry of the lattice. Our finding of "hexagonal" tubes is in contrast to previous observations of tubes with almost circular cross sections [15]. The question then arises about the lowest energy structure of the lattice of aligned tubes and the possibility of having several metastable structures depending on the growth conditions. To gain some insight into these problems, we have performed an extensive search of possible metastable structures of the (triangular) lattice of aligned tubes. Eventually, the equilibrium configuration of the lattice will be also found.

It is well established that freestanding isolated tubes present circular cross sections since the circular shape minimizes the strain energy of the tubes. (In this context, the strain energy is the elastic energy needed to roll up a graphene sheet into a cylinder to form a nanotube.) Fig. 1 shows the strain energy of armchair and zigzag SWCNTs as a function of tube diameter $D$. This energy decreases as $1 / R^{2}$ ( $R$ is the tube radius) as expected from the continuum elastic model [16]. The tubes, however, may deform elastically when they interact either with a substrate or

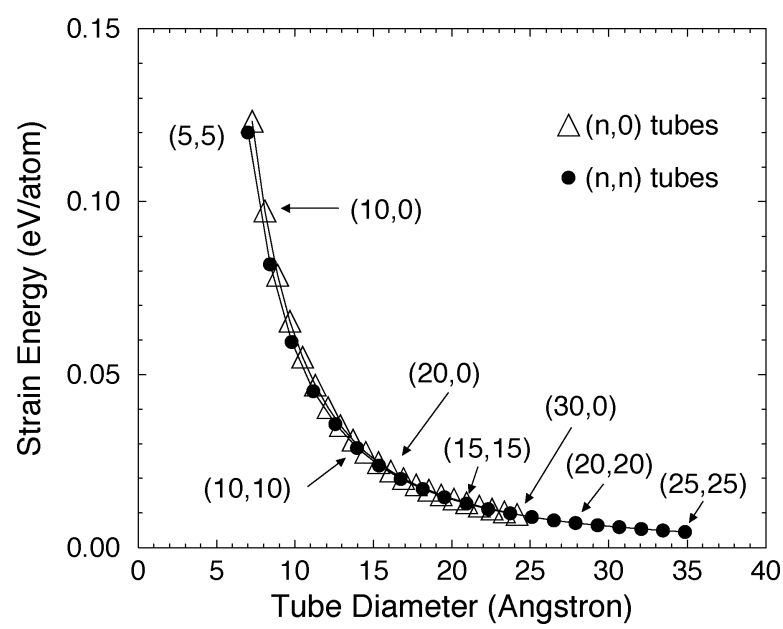

Fig. 1. Strain energy of armchair $(n, n)$ and zigzag $(n, 0)$ SWCNTs as a function of tube diameter.

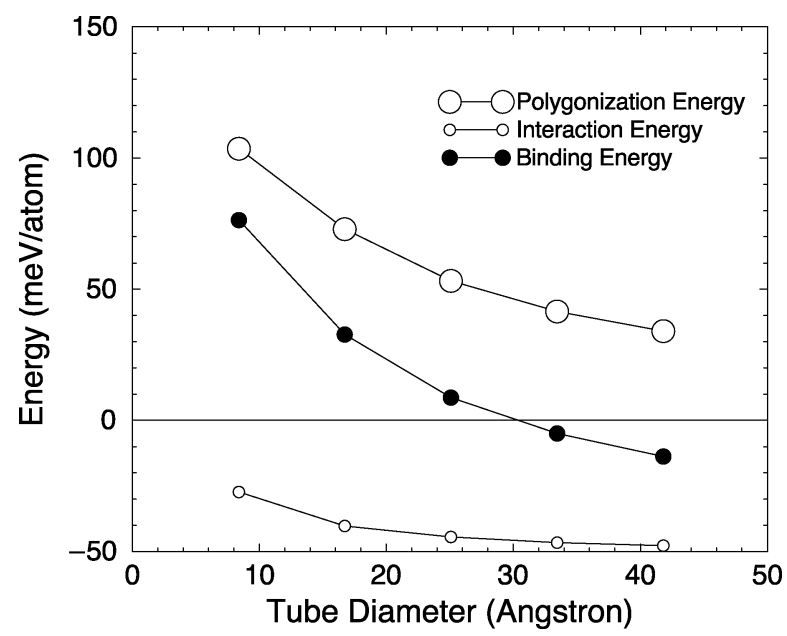

Fig. 2. Cohesive energy, with respect to the isolated circular tubes, and intertube interaction energy of a lattice of hexagonal $(n, n)$ tubes as a function of tube diameter. Also shown is the polygonization energy of the $(n, n)$ hexagonal tubes (see text for details).

with other tubes. Therefore, tubes in a bundle may depart, due to the intertube van der Waals-type interaction, from the circular shape. The deformation energy, i.e., energy cost of changing the cross section of the tubes from the ideal circular shape, should then be compensated by the intertube interaction energy, which acts as the driving force for the elastic structural changes of the tubes. Fig. 2 shows the deformation (polygonization) energy of armchair hexagonal tubes, intertube interaction energy between those tubes when forming a triangular lattice, and cohesive energy of that lattice of hexagonal tubes with respect to the isolated circular tubes, all as functions of tube diameter $D$. The balance between the deformation energy, which opposes the polygonization of the tubes in the lattice, and the intertube interaction energy yields to stable lattices of hexagonal armchair tubes for tube diameters larger than $30 \AA$.

The lattice of circular tubes is always a bound system irrespective of the diameter of the constituent tubes (see Fig. 3). Its cohesive energy, however, is pretty small, as compared to the interaction energy between graphene layers in graphite. The 


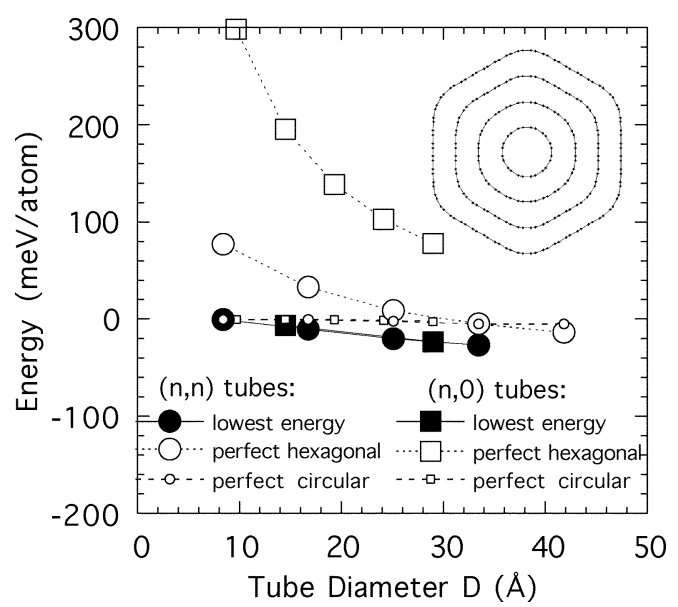

Fig. 3. Cohesive energy with respect to the isolated circular tubes of the triangular lattice of: 1) circular, 2) hexagonal, and 3) lowest energy tubes as a function of tube diameter. $(n, n)$ armchair and $(n, 0)$ zigzag tubes are considered. The inset shows, superimposed, the structures of the lowest energy $(n, n)$ tubes.

reason being in the lack of planar interacting faces between adjacent tubes. Notice that the interaction energy between hexagonal tubes, which exhibit planar facets lying parallel one to another between adjacent tubes, is one order of magnitude larger than that of the lattice of circular tubes (see Fig. 2). However, for small diameter tubes, this energy does not compensate the high deformation energy of hexagonal tubes and the lattice of circular tubes is more stable than the corresponding lattice of hexagonal tubes. Eventually, since the deformation energy decreases with increasing tube diameter, the lattice of hexagonal tubes becomes more stable than the lattice of circular tubes (see Fig. 3). The onset of polygonization, $D=34$ and $50 \AA$ for armchair and zigzag tubes, respectively, is, however, too high as compared with the experimental value $D=17 \AA$.

To obtain metastable, and eventually the lowest energy, structures of the lattice of tubes, we have applied the thermal quenching procedure. First, a number of lattice configurations corresponding to several values of the lattice parameter and to tube shapes ranging from circular to hexagonal are generated. All those high energy configurations of the lattice are then cooled down into rigid structures corresponding to local minima of the potential energy surface. The relaxations yield several metastable structures characterized by different tube cross sections and unit cell volume. The shape of the tubes changes, with increasing cell volume, from "nearly" hexagonal to "almost" circular, going through an intermediate volume lattice formed by hexagonal tubes with rounded corners. The lowest energy configuration of the lattice evolves from circular to hexagonal tubes with increasing tube diameter. For $D \leq 17 \AA$, nearly circular tubes are found. This explains why all previous observations of bundles of tubes in this size range show tubes with circular cross sections.

However, the metastable structure, shown in Fig. 4, formed by rounded hexagonal tubes is only $0.6 \mathrm{meV} /$ atom above the circular lowest energy structure of $(12,12)$ tubes. Similarly, the metastable structure of rounded hexagonal $(18,0)$ tubes is only $2.8 \mathrm{meV} /$ atom above the minimum. These novel metastable structures give support to the crystalline bundles of polygonized tubes found in the experiment.

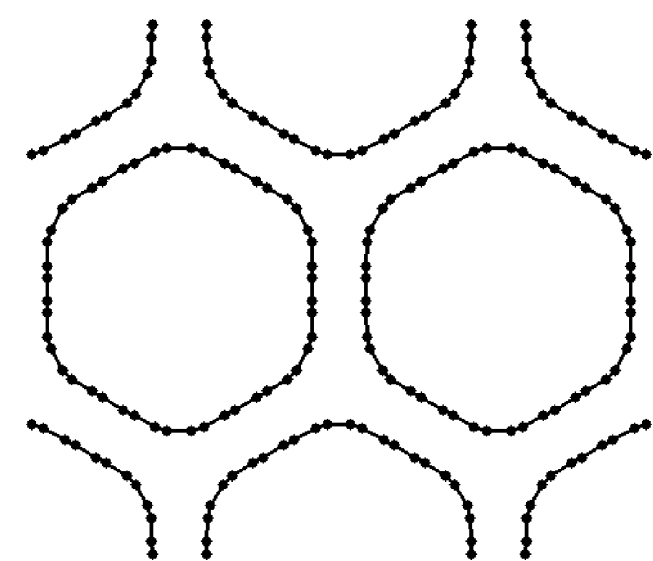

Fig. 4. Metastable structure of a lattice of $(12,12)$ tubes with rounded hexagonal cross section. This structure supports the novel crystalline ropes of polygonized tubes produced by $\mathrm{CO}_{2}$ laser ablation.

\section{THERMAL TRANSFORMATIONS OF SWCNT ROPES}

Coalescence of two SWCNTs into a larger diameter SWCNT has been observed upon the annealing of SWCNTs at high temperatures either in the presence of $\mathrm{H}_{2}$ [17] or under electron irradiation [18]. We have discovered [11], as we were investigating the stability of SWCNT ropes under thermal treatment, a new structural transformation of bundles of SWCNTs into MWCNTs. Samples 1 of SWCNT ropes produced by the catalytic arc-discharge technique [4] were heated for approximately $15 \mathrm{~min}$ at temperatures between $1600{ }^{\circ} \mathrm{C}-2800{ }^{\circ} \mathrm{C}$ under argon flow. Above $2200{ }^{\circ} \mathrm{C}$, the bundles disappear, giving rise to MWCNTs consisting of 2-6 nested tubes. All the characterization techniques of the samples, i.e., HRTEM, X-ray diffraction, and Raman spectroscopy, confirm the structural reorganization of the bundles. This single-wall (SW)-to-multiple-wall (MW) transformation is also confirmed by extensive MD simulations (see below). The simulations combined with the experimental results unveil the physical "patching-and-tearing" mechanism of this showy transformation. This novel mechanism is of general applicability and describes both the coalescence of two SWCNTs into a larger diameter SWCNT and the newest transformation of SWCNT ropes into MWCNTs.

\section{A. Coalescence of Two Tubes}

We first study the temperature-induced coalescence of two tubes. The presence of defects in the tubes may act as a driving force for coalescence. It has been shown that vacancies are the leading defects promoting coalescence [18]. Vacancies can be produced by chemical treatment [17], electron irradiation [18], and by thermal treatment of the tubes at relatively high temperatures (above $1600{ }^{\circ} \mathrm{C}$ ). The number of vacancies produced by the thermal treatment clearly depends on the vacancy formation energy and on the temperature and duration of the heat treatment. However, a trustworthy evaluation of the concentration of vacancies is not possible from the experimental data at hand, especially since the vacancies are not generated under

${ }^{1}$ HRTEM characterization of the samples shows that they are made of bundles of SWCNTs with bundle diameters ranging from 5 to $20 \mathrm{~nm}$. The individual tubes of approximately $1.4 \mathrm{~nm}$ in diameter are arranged in a triangular lattice. 


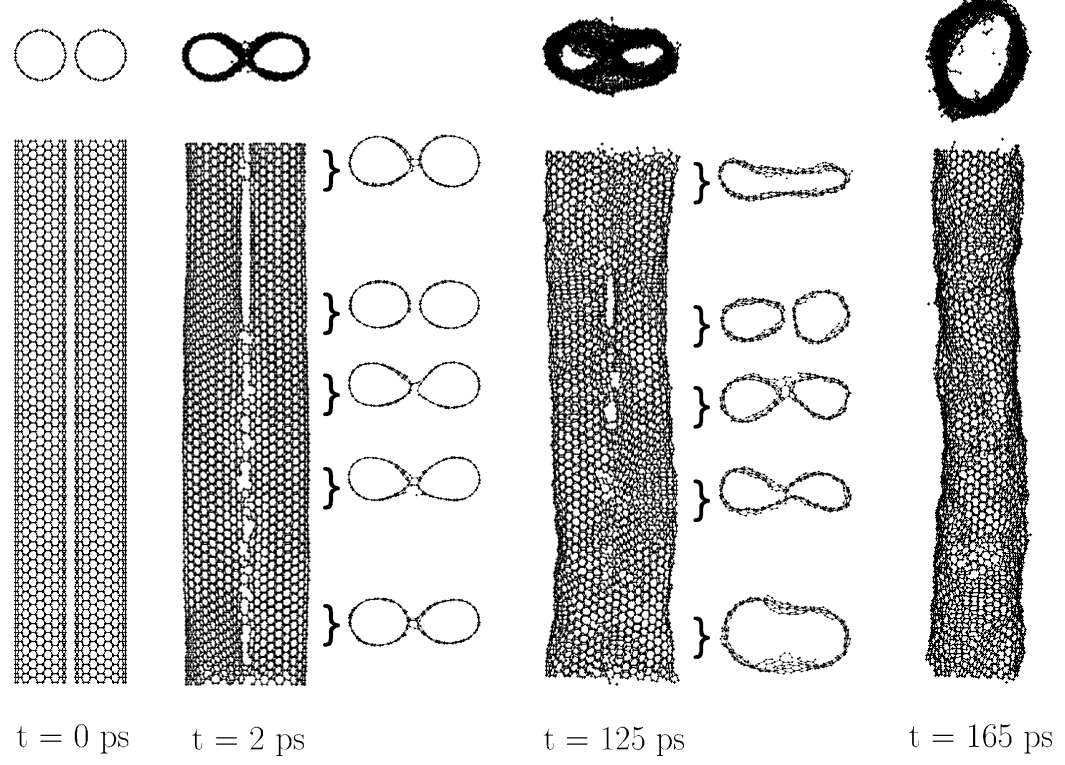

Fig. 5. Snapshots (side and top views) showing the sequence of the coalescence of two $(10,10)$ tubes. Also shown are top views of some tube portions embraced by brackets.

equilibrium conditions. For long enough (in this case, approximately minutes [11]) heat treatments, though, a rough estimate is given by the inverse of the number of atoms required to sum up a vibrational energy of the order of (two or three times) the vacancy formation energy. Since the vacancy formation energy in a $(10,10)$ tube is approximately $14 \mathrm{eV}$, the estimated concentration of vacancies produced by the heat treatment at $2500 \mathrm{~K}$ is of $1.1 \%-0.7 \%$. Due to the high temperatures, the vacancies have a high mobility and diffuse throughout the tube until they get locked in the intertube region by saturation of the associated dangling bonds with the dangling bonds left free by a nearby vacancy in the adjacent tube. Saturation of dangling bonds between neighboring tubes yields to intertube polymerization, which is the initial stage for the coalescence of tubes. At the experimental temperatures, the creation of vacancies in the tubes requires heat treatments of approximately minutes. This time scale is clearly not tractable in conventional MD simulations. However, once a sufficient number of intertube links are formed, coalescence proceeds within a time scale of a few hundred picoseconds. It is this second part of the process that is suitable for being simulated in the computer and, therefore, we concentrate our study in this part.

We have simulated the coalescence of two $(10,10)$ tubes under thermal treatment (see Fig. 5). The initial intertube distance between the tubes is of $17 \AA$. The first step consists of the generation of vacancies by random removal of a number $(2 \%)$ of atoms. The choice of a simulated concentration of vacancies of $2 \%$ has been guided by the value $(1.1 \%-0.7 \%)$ estimated for the experiments. We have checked that the larger concentration of vacancies used in the dynamical simulations has the only effect of reducing the time scale for coalescence without modifying its mechanism. Since no significant diffusion of vacancies is expected within the time scale of the simulations, only those vacancies in the vicinity of the partner tube play an active role in the coalescence process. Therefore we restrict the removal of atoms to the intertube region. Moreover, long simulation cells, containing 60 unit cells in the axial direction of the tubes, are considered to avoid unphysical uniform distributions of vacancies arising from the use of periodic boundary conditions in the axial direction of the tubes. Polymerization of the two tubes occurs very rapidly (in $2 \mathrm{ps}$ ) after the generation of vacancies. The formation of intertube links is driven by the saturation of the dangling bonds left free by the vacancies, which results in a nonuniform distribution of the links along the tubes length (see Fig. 5, $t=2 \mathrm{ps}$ ). By progressively heating up the tubes, the initial intertube links develop into enveloping surfaces (or "patches") common to the two tubes. The "tearing" apart of the intratube bonds in the intertube region in some of the patches gives rise to partial coalescence of the tubes, whereas other portions of the tubes remain polymerized or even completely unlinked (see Fig. $5, t=125 \mathrm{ps}$ ). As the time evolves, new intertube links and patches develop, the existing patches and the coalesced parts of the tubes grow in the axial direction and merge one to another, until eventually, all the remaining intratube bonds in the intertube region "tear" apart, producing the coalesce of the two tubes in their whole length (see Fig. 5, $t=165 \mathrm{ps}$ ). The patching-and-tearing mechanism emerging from our simulations explains the coalescence of two tubes in great detail. This mechanism gives support to the early intuitive "zipping" description of coalescence [17]. Moreover, our results on the coalescence of two $(10,10)$ tubes are in fair agreement with the tight-binding simulations of Terrones et al. [18]. We have also found (in contrast to the earlier statement [18] that this process was very unlikely) that coalescence proceeds between tubes of different chiralities without any restriction. Fig. 6 shows the coalescence of a $(10,10)$ and a $(12,8)$ tubes. The two tubes have similar radii and a long simulation cell containing approximately (due to the incommensurability of the two tubes) 68 and 9 unit cells, respectively, has been used in the simulations. We find coalescence of the tubes even in the most stringent case of two nonchiral tubes, one of the armchair type, the $(10,10)$ 

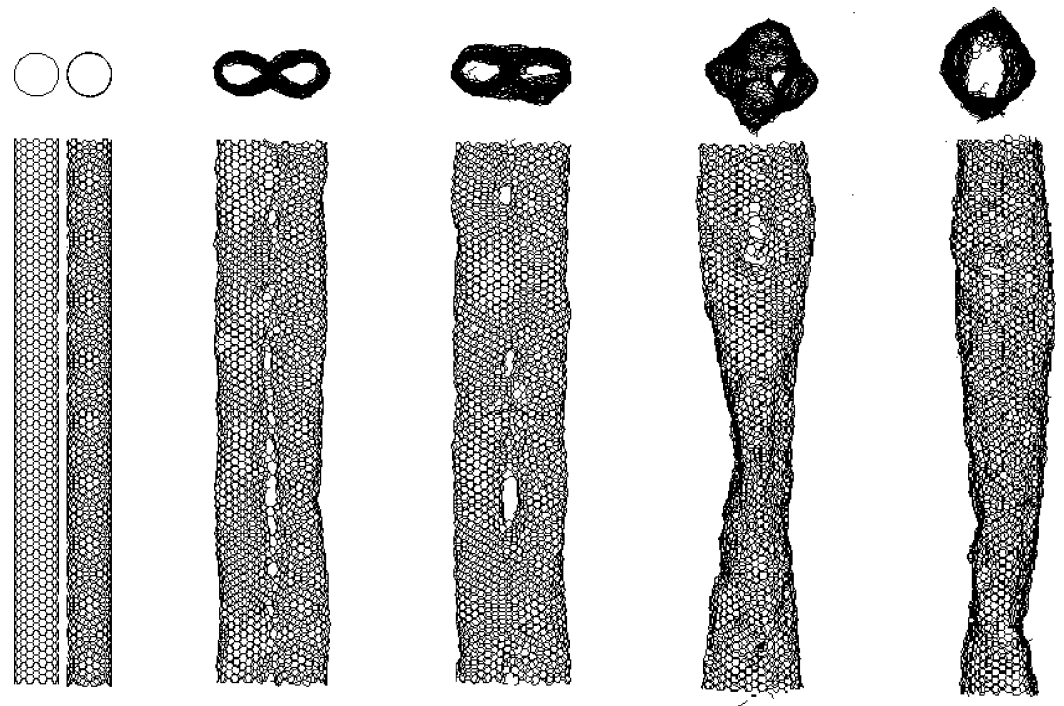

Fig. 6. Snapshots (side and top views) showing the sequence of the coalescence of a $(10,10)$ and a $(12,8)$ tubes.

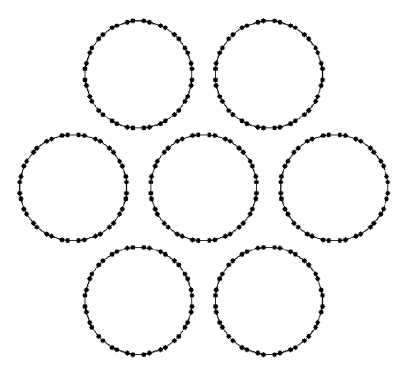

$\mathrm{t}=0 \mathrm{ps}$

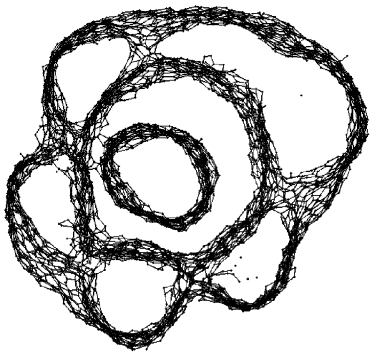

$\mathrm{t}=213 \mathrm{ps}$

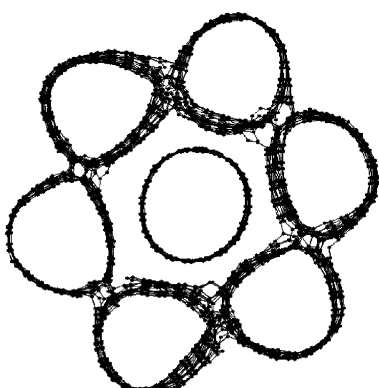

$\mathrm{t}=1 \mathrm{ps}$

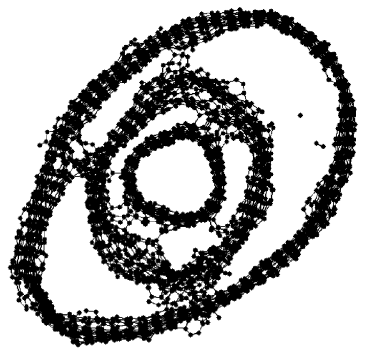

$\mathrm{t}=245 \mathrm{ps}$

Fig. 7. Snapshots (top views) taken from the simulations showing the sequence of the SW-to-MW transformation of a bundle of seven $(10,10)$ tubes.

tube, and the other one of the zigzag type, the $(17,0)$ tube. For both pairs of tubes, large tube reconstructions are required for knitting the two corresponding tubes into a larger diameter SWCNT due to the mismatch of their respective honeycomb structures. The extent of reconstruction required can only be accomplished by using long simulation cells as the ones used in the two examples presented in this paper. In summary, we have demonstrated that no restrictions apply for the coalescence of tubes of different chiralities provided that the simulation cell in the axial direction of the tubes is long enough. Coalescence proceeds following the same patching-and-tearing mechanism introduced to explain coalescence of nonchiral tubes. However,<smiles>C1CCC2CCCCC3CCCCCC34CCCCC24CC1</smiles>

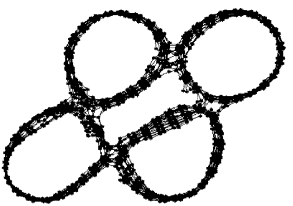

$\mathrm{t}=0 \mathrm{ps}$

$\mathrm{t}=1 \mathrm{ps}$
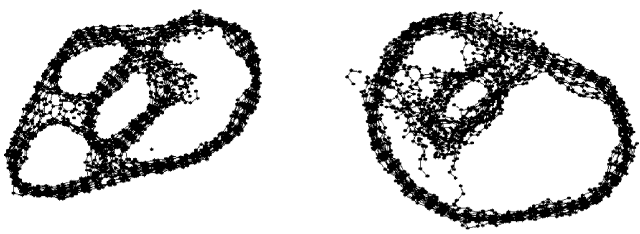

$\mathrm{t}=970 \mathrm{ps}$

$\mathrm{t}=1030 \mathrm{ps}$

Fig. 8. Snapshots (top views) taken from the simulations showing the transformation of a bundle of four $(10,10)$ tubes into an MWCNT consisting on two nested tubes.

it is fair to recognize that the resulting tubes have higher concentrations of defects than in the case of coalescence of nonchiral tubes of the same type. Little or no annealing of those defects is observed within the limited time scale of our simulations.

As we have pointed out above, large simulation cells are used to avoid unphysical uniform distributions of vacancies arising from the repetition of the cell within the periodic boundary conditions scheme and to accommodate the tube reconstruction involved in the coalescence of tubes with different chiralities. However, the use of smaller cells would be preferred from the computational (time saving) view point. We have studied the effect of reducing the size of the simulation cell down to 20 unit cells in the axial direction of the tubes in the case of coalescence of two nonchiral $(10,10)$ tubes. The use of the smaller cell does not change the outcome of the simulated coalescence, and the patching-and-tearing mechanism describing the process 

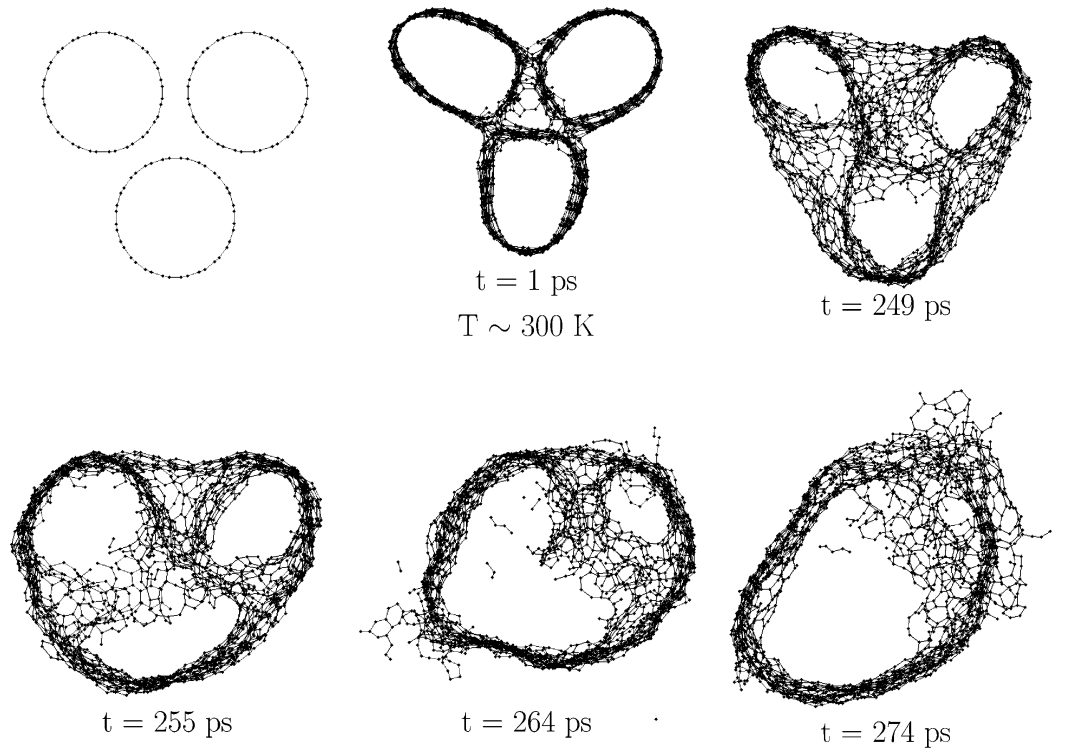

Fig. 9. Snapshots (top views) taken from the simulations showing the coalescence of a bundle of three $(10,10)$ tubes into a larger diameter SWCNT.

remains valid. Therefore, in Section IV-B, where we simulate bundles of $(10,10)$ tubes, we will use the smaller simulation cell without any loss of generality or physical insight.

\section{B. Transformation of SWCNTs Ropes Into MWCNTs}

Let us first consider a bundle of seven $(10,10)$ tubes arranged in a triangular lattice (with a lattice parameter of $17 \AA$ ) with a central tube and six surrounding tubes (see Fig. 7). For investigating the temperature-induced SW-to-MW transformation, we assume that, similar to the case of coalescence of two tubes, the vacancies play a leading role in promoting the structural reorganization of the bundles. We will also consider in the simulations a larger $(4 \%)$ concentration of vacancies than the experimental estimate to speed up the transformation process, assuming that, as in the case of coalescence, this larger concentration does not change its mechanism. These assumptions will be lately confirmed by the simulations (see below). The thermal treatment of the bundle at elevated temperatures will produce a number of vacancies in the tubes. Therefore, we begin the simulation by generating a number (4\%) of vacancies in the intertube regions defined by the six outer tubes (no defects are created in the central tube, see below for a justification). Upon the generation of vacancies, polymerization between neighboring tubes takes place in less than 1 ps (see Fig. 7, $t=1 \mathrm{ps}$ ). The bundle is then progressively heated up. Coalescence between neighboring tubes begins to develop following the patching-and-tearing mechanism described above (see Fig. 7, $t=213 \mathrm{ps)}$ ). The concerted coalescence of the tubes around the bundle gives rise to the transformation of the bundle of SWCNTs into an MWCNT consisting of three nested tubes (see Fig. 7, $t=245 \mathrm{ps}$ ). The central tube may also have a number of defects. Depending on the links established between the central tube and its surroundings, three (such as the one shown here) or two nested structures may then occur.

Bundles containing four and five tubes transform into MWCNTs consisting of two nested tubes (see Fig. 8) following a similar patching-and-tearing mechanism operating in a concerted way around the bundle. We find that four tubes in the bundle is the lower limit to observe the SW-to-MW transformation. Thus, the concerted coalescence of three $(10,10)$ SWCNTs produces a SWCNT of larger diameter (see Fig. 9). One could argue about the small amount of $\mathrm{C}$ atoms available to reconstruct an internal tube. However, we find that a bundle of three $(20,20)$ tubes (where the supply of $\mathrm{C}$ atoms would be sufficient to reconstruct an internal $(9,9)$ tube) also coalesces in a SWCNT of larger diameter.

The excellent agreement between the simulations and experiments gives support for the assumptions made about the role played by the vacancies in the SW-to-MW transformation. We want to also stress that the patching-and-tearing mechanism presented here is of general applicability to describe both the coalescence of SWCNTs into a larger diameter tube and the newest SW-to-MW transformation based on the concerted coalescence of the tubes in a bundle.

\section{SUMmary}

Novel crystalline ropes of polygonized SWCNTs (tube diameter $D$ approximately $17 \AA$ ) produced by $\mathrm{CO}_{2}$ laser ablation exhibit rounded-hexagonal cross sections. Simulations of the lattice show several metastable structures characterized by different tube cross sections, hexagonal, rounded-hexagonal and circular, and increasing cell volume. The lowest energy configuration of the lattice progresses from circular to hexagonal with increasing tube diameter. The driving force for the departure of the tubes from the ideal circular shape is the attractive (van der Waals type) intertube interaction. In contrast to the experimental ropes, the lowest energy configuration of the lattice of tubes with $D=17 \AA$ corresponds to nearly circular tubes. However, the lattice of rounded-hexagonal tubes is very close in energy, which would explain the experimental observation. It would be of great interest to understand and control the production of the different metastable structures of the lattice depending on the growth conditions. 
The experimental observation of the transformation of SWCNT bundles into MWCNTs under high-temperature treatments motivated us to simulate the thermal stability of the ropes. Extensive MD simulations combined with the experimental results unveil the physical mechanism of the SW-to-MW transformation based on the patching of SWCNTs and their subsequent tearing apart, giving rise to an MWCNT. The driving force for this transformation are the vacancies created by the high-temperature treatment. Further studies are required to improve our understanding of the structural stability of the ropes and their possible morphological changes. Possible implications on the different growth regimes of SWCNT bundles are still obscure.

\section{ACKNOWLEDGMENT}

The study was performed in collaboration with the groups of S. Iijima, NEC Corporation, Tsukuba, Japan, and Meijo University, Nagoya, Japan, and S. Bonamy, Centre National de la Recherche Scientifique (CNRS) Universite, Orelans, France.

\section{REFERENCES}

[1] S. Iijima and T. Ichihashi, "Single-shell carbon nanotubes of 1-nm diameter," Nature, vol. 363, pp. 603-605, 1993.

[2] S. Iijima, "Helical microtubules of graphitic carbon," Nature, vol. 354, pp. 56-58, 1991.

[3] A. Thess, R. Lee, P. Nikolaev, H. Dai, P. Petit, J. Robert, C. Xu, Y. H. Lee, S. G. Kim, D. Colbert, G. Scuseria, D. Tomanek, J. Fischer, and R. Smalley, "Crystalline ropes of metallic carbon nanotubes," Science, vol. 273, pp. 483-487, 1996.

[4] C. Journet, W. K. Maser, P. Bernier, A. Loiseau, M. L. de la Chapelle, S. Lefrant, P. Deniard, R. Lee, and J. E. Fischer, "Large-scale production of single-walled carbon nanotubes by the electric-arc technique," Nature, vol. 388, pp. 756-758, 1997.

[5] T. Hertel, R. E. Walkup, and P. Avouris, "Deformation of carbon nanotubes by surface van der Waals forces," Phys. Rev. B, Condens. Matter, vol. 58, pp. 13870-13873, 1998 .

[6] R. S. Ruoff, J. Tersoff, D. C. L. S. Subramoney, and B. Chan, "Radial deformation of carbon nanotubes by van der Waals forces," Nature, vol. 364, pp. 514-516, 1993.

[7] J. Tersoff and R. S. Ruoff, "Structural properties of a carbon-nanotube crystal," Phys. Rev. Lett., vol. 73, pp. 676-679, 1994.

[8] M. J. López, A. Rubio, J. A. Alonso, L.-C. Qin, and S. Iijima, "Novel polygonized single-wall carbon nanotube bundles," Phys. Rev. Lett., vol. 86, pp. 3056-3059, 2001.

[9] M. L. de la Chapelle, S. Lefrant, C. Journet, W. K. Maser, P. Bernier, and A. Loiseau, Molecular Nanostructures, H. Kuzmany, J. Fink, M. Mehring, and S. Roth, Eds, Singapore: World Sci., 1998, p. 387.

[10] K. Méténier, S. Bonnamy, F. Béguin, C. Journet, P. Bernier, M. L. de la Chapelle, O. Chauvet, and S. Lefrant, "Coalescence of single-walled carbon nanotubes and formation of multi-walled carbon nanotubes under high-temperature treatments," Carbon, vol. 40, pp. 1765-1773, 2002.

[11] M. J. López, A. Rubio, J. A. Alonso, S. Lefrant, K. Méténier, and S. Bonnamy, "Patching and tearing single wall carbon-nanotube ropes into multiwall carbon nanotubes," Phys. Rev. Lett., vol. 89, pp. 255 501-1-255 501-4, 2002.

[12] J. Tersoff, "New empirical approach for the structure and energy of covalent systems," Phys Rev. B, Condens. Matter, vol. 37, pp. 6991-7000, 1988.

[13] — -Empirical interatomic potential for carbon, with applications to amorphous carbon," Phys. Rev. Lett., vol. 61, pp. 2879-2882, 1988.

[14] K. Nordlund, J. Keinonen, and T. Mattila, "Formation of ion irradiation induced small-scale defects on graphite surfaces," Phys. Rev. Lett., vol. 77, pp. 699-702, 1996

[15] L.-C. Qin and S. Iijima, "Structure and formation of raft-like bundles of single-walled helical carbon nanotubes produced by laser evaporation," Chem. Phys. Lett., vol. 269, pp. 65-71, 1997.

[16] G. G. Tibbetts, "Why are carbon filaments tubular?," J. Cryst. Growth, vol. 66, pp. 632-638, 1984
[17] P. Nikolaev, A. Thess, A. G. Rinzler, D. T. Colbert, and R. E. Smalley, "Diameter doubling of single-wall nanotubes," Chem. Phys. Lett., vol. 266, pp. 422-426, 1997.

[18] M. Terrones, H. Terrones, F. Banhart, J.-C. Charlier, and P. M. Ajayan, "Coalescence of single-walled carbon nanotubes," Science, vol. 288, pp. $1226-1229,2000$.

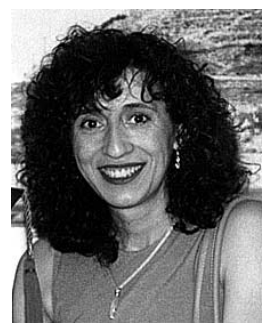

María J. López received the Ph.D. degree in physics from the University of Valladolid, Valladolid, Spain, in 1990.

From 1991 to 1993, she was a Post-Doctoral Fulbright Fellow with the Argonne National Laboratory, where she was involved with phase transitions and fragmentation on finite systems (atomic clusters) and developed interatomic potentials for transition and noble metals. From 1993 to 2001, she was an Assistant Professor and then Research Associate with the University of Valladolid. Since 2001, she has been a Ramón y Cajal Research Associate with the University of Valladolid. She has visited several universities, including the École Polytechnique Federal de Lausanne, Lausanne, Switzerland, Freie Universität Berlin, Germany, and the Institute for Nuclear Theory (INT), University of Washington, Seattle. Her current research focuses on nanotechnology including clusters, CNTs, and assembled materials.

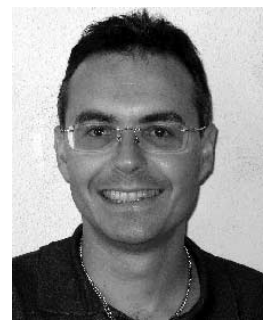

Angel Rubio was born in 1965 . He received the $\mathrm{Ph} . \mathrm{D}$. degree in physics from the University of Valladolid, Valladolid, Spain, in 1991.

From 1993 to 2001, he was an Associate Professor of theoretical physics with the University of Valladolid. He is currently a Full Professor of condensed matter physics with the Facultad de Qumicas, Departamento de Física de Materiales, Centro Mixto CSIC Universidad del País (UPV)/EHU and the Donostia International Physics Center (DIPC), San Sebastián/Donostia, Spain. He has been a Visiting Professor with different universities including the University of California at Berkeley, the École Polytechnique, Paris, France, and the Max-Planck-Institut Berlin, Berlin, Germany. Since 2001, he has helped established the DIPC, a multidisciplinary center on computational material science and a nanotechnology center of excellence. He has authored or coauthored over 100 publications appearing in scientific journals, with over 2500 total citations. $\mathrm{He}$ has edited two books about nanotechnology. He holds one patent.

Dr. Rubio has contributed two reviews of modern physics. He has presented over 60 invited talks at international conference and meetings. He is the co-director of several national and international grants for research in nanostructures. $\mathrm{He}$ is a spokesperson of the Nanostructures and Nanotechnologies Working Group of the psi-k Network (European Science Foundation Programme Electronic Structure Calculations for Elucidating the Complex Atomistic Behavior of Solids and Surfaces). He was the recipient of the Royal Spanish Physical Society Prize "Jovenes Investigadores," the Honor Prize for his Ph.D. thesis in physics, and the National Prize for graduates in physics.

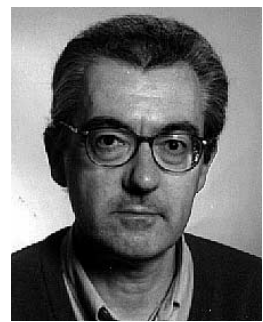

Julio A. Alonso was born in 1948. He received the $\mathrm{Ph} . \mathrm{D}$. degree in physics from the University of Valladolid, Valladolid, Spain, in 1975.

From 1975 to 1980 , he was a Post-Doctoral Fellow and Research Associate with the Laboratory for Research on the Structure of Matter, University of Pennsylvania, where he was involved with the theory of chemical binding in metallic alloys and also with the development of nonlocal approximations to exchange and correlation in density functional theory. From 1980 to 1988 , he was an Associate Professor with the University of Valladolid. Since 1988, he has been a Professor of physics with the University of Valladolid. He has been a Visiting Scientist spending sabbatical years with the University of East Anglia, Norwich, U.K. and the Donostia International Physics Center, San Sebastian, Spain. He has also spent extended periods with the University of Osnabrïck, Osnabrück, Germany, International Center for Theoretical Physics, Trieste, Italy, and Queens University at Kingston, Kingston, ON, Canada. His research interests include density functional theory, amorphous alloys, atomic clusters, and CNTs. 